\title{
Chloride Channel and Inflammation-Mediated Pathogenesis of Osteoarthritis
}

\author{
Zicong Lin ${ }^{1} *$, Zhiqin Deng $\mathbb{D}^{1, *}$, Jianquan Liu ${ }^{1}, *$, Zhongshi Lin², Siyu Chen ${ }^{3}$, Zhenhan Deng $\mathbb{D}^{3}$, \\ Wencui $\mathrm{Li}^{\mathrm{I}}$
}

'Hand and Foot Surgery Department, The First Affiliated Hospital of Shenzhen University, Shenzhen Second People's Hospital, Shenzhen, Guangdong, 518035, People's Republic of China; ${ }^{2}$ Shenzhen Institute for Drug Control (Shenzhen Testing Center of Medical Devices), Shenzhen, Guangdong, 5 18057, People's Republic of China; ${ }^{3}$ Department of Sports Medicine, The First Affiliated Hospital of Shenzhen University, Shenzhen Second People's Hospital, Shenzhen, Guangdong, 518035, People’s Republic of China

*These authors contributed equally to this work

Correspondence: Zhenhan Deng, Department of Sports Medicine, The First Affiliated Hospital of Shenzhen University, Shenzhen Second People's Hospital, 3002 Sungang West Road, Shenzhen City, 5I8025, People's Republic of China, Tel +86 I3928440786, Fax +86 755-83366388, Email dengzhenhan@email.szu.edu.cn; Wencui Li, Department of Hand and Foot Surgery, The First Affiliated Hospital of Shenzhen University, Shenzhen Second People's Hospital, 3002 Sungang West Road, Shenzhen City, 5I8025, People's Republic of China, Tel +86 I3923750767, Email liwencui@email.szu.edu.cn

\begin{abstract}
Articular cartilage allows the human body to buffer and absorb stress during normal exercise. It is mainly composed of cartilage cells and the extracellular matrix and is surrounded by the extracellular microenvironment formed by synovial fluid and various factors in it. Studies have shown that chondrocytes are the metabolic center of articular cartilage. Under physiological conditions, the extracellular matrix is in a dynamic balance of anabolism and catabolism, and various factors and physical and chemical conditions in the extracellular microenvironment are also in a steady state. This homeostasis depends on the normal function of proteins represented by various ion channels on chondrocytes. In mammalian chondrocyte species, ion channels are mainly divided into two categories: cation channels and anion channels. Anion channels such as chloride channels have become hot research topics in recent years. These channels play an extremely important role in various physiological processes. Recently, a growing body of evidence has shown that many pathological processes, abnormal concentration of mechanical stress and chloride channel dysfunction in articular cartilage lead to microenvironment disorders, matrix and bone metabolism imbalances, which cause partial aseptic inflammation. These pathological processes initiate extracellular matrix degradation, abnormal chondrocyte death, hyperplasia of inflammatory synovium and bony. Osteoarthritis (OA) is a common clinical disease in orthopedics. Its typical manifestations are joint inflammation and pain caused by articular cartilage degeneration, but its pathogenesis has not been fully elucidated. Focusing on the physiological functions and pathological changes of chloride channels and pathophysiology of aseptic inflammation furthers the understanding of OA pathogenesis and provides possible targets for subsequent medication development.
\end{abstract}

Keywords: osteoarthritis, chondrocyte, ion channel, inflammatory factors

\section{Introduction}

\section{Articular Cartilage}

In the healthy human body, articular cartilage covers the opposing bone surface between adjacent long bones and is an important part of joints. Articular cartilage provides a smooth contact surface between bones, and due to the elasticity and deformability of cartilage itself, it can cushion powerful vibrations and shock loads during joint movement, thus facilitating safe and painless movement in healthy human body.

Anatomical studies have shown that articular cartilage is composed of many non-cellular components and a few types of cartilage cells located in the cartilage lacuna. ${ }^{1,2}$ Cartilage cells, or chondrocytes, are the metabolic center of articular cartilage. To withstand the extreme stress load during exercise, chondrocytes synthesize and secrete copious amounts of extracellular matrix, which is essential for a healthy joint cavity microenvironment. ${ }^{3}$ The extracellular matrix of articular 
cartilage is mainly composed of type II collagen fibers interlaced with each other, while other types of collagen fibers such as VI, IX and XI are also involved in its formation. ${ }^{1,4-6}$ For this reason, in the pathological process of cartilage degeneration, a change in type II collagen content is important and is also a key factor in basic research. The noncollagen components in the extracellular matrix mainly include anchoring proteins, which help chondrocytes become fixed in the cartilage lacuna, and a large amount of aggrecan, which helps chondrocytes resist stress loads during exercise. $^{3}$

Cartilaginous lesions are accompanied by abnormal changes in cells and/or changes in the extracellular matrix and environment. Therefore, the author will focus on cartilage cells and review recent research progress on osteoarthritis $(\mathrm{OA})$.

\section{Osteoarthritis}

$\mathrm{OA}$ is a chronic disease of the motor system caused by many factors including advanced age, reduced estrogen and trauma. It is the second leading cause of dyskinesia in adults, mainly manifested as pain in the affected joint, degeneration of articular cartilage, osteophyte hyperplasia and sclerosis of subchondral bone. ${ }^{7}$ OA affects $>250$ million people worldwide. ${ }^{8}$ Most patients with OA have varying degrees of activity restriction and a considerable number eventually develop disabilities. ${ }^{7,9,10}$ Every year, $>400,000$ patients with OA receive joint replacement surgery, the radical treatment for end-stage OA. Patients with OA experience a great financial burden from medical expenses related to its treatment. ${ }^{9}$

The most important articular manifestation of OA is cartilage degeneration or cartilage destruction, in addition to other intra-articular or extra-articular diseases such as bone hyperplasia, joint fluid changes, subchondral bone sclerosis and inflammatory slip. Moreover, OA often leads to chronic pain, which is caused by factors such as inflammation. However, some studies have shown that calcium ion-activated chloride channels can regulate the excitability of neurons, and this feature may be involved in the generation, development and maintenance of acute or chronic pain. ${ }^{11}$ In related animal experiments, this conclusion has also been verified to a certain extent. ${ }^{12}$

Academia has not arrived at a definite consensus on the pathogenesis of OA. Therefore, theories on the mechanism of $\mathrm{OA}$ involve various disease processes. In recent years, several remarkable theories on either a single aspect of OA or multiple, interactive mechanisms of pathology have emerged, including those involving changes in the extracellular microenvironment, abnormal extracellular matrix metabolism, cellular inflammatory response and abnormal cell death. One kind of theory involves the role of the chloride channels in OA, and many researchers have demonstrated that chloride channels participate in OA pathogenesis.

\section{Chloride Channels and OA}

In the process of articular cartilage cell response to various stimuli, ion channels (including cation channels and anion channels) are considered to be important nodes that regulate cartilage cell function. ${ }^{3}$ Among them, the chloride $\left(\mathrm{Cl}^{-}\right)$ channel is an important part of the anion channel, and it plays an irreplaceable biological function. Generally, $\mathrm{Cl}^{-}$ channels are not one protein or a kind of protein, but a family of proteins that perform physiological functions through different mechanisms, including voltage-dependent $\mathrm{Cl}^{-}$channels $(\mathrm{ClC})$, cystic fibrosis transmembrane conductance regulator channels (CFTR), swelling-activated $\mathrm{Cl}^{-}$channels, $\mathrm{Ca}^{2+}$-activated $\mathrm{Cl}^{-}$channels and coordination gatedependent ligand-gated $\mathrm{Cl}^{-}$channels. ${ }^{3,13}$ These $\mathrm{Cl}^{-}$channels are believed to be involved in important physiological activities such as regulating ion homeostasis, maintaining cell excitability and muscle tension, mediating nerve signal or hormone transmission and material transport and regulating cell volume and survival. ${ }^{14-18}$

In recent years, more and more studies have turned their attention to this field, in an attempt to find the potential relationship or mechanism between the chloride channel and OA. And as mentioned above, the $\mathrm{Cl}^{-}$channels distributed on chondrocytes are involved in the regulation and progress of various physiological activities. After summarizing recent literatures and our experimental results, we believe $\mathrm{Cl}^{-}$channels play an imperative role in pathogenesis of OA, including changes of extracellular microenvironment, abnormal metabolism of extracellular matrix, inflammation and abnormal cell death. These studies have demonstrated some specific mechanism of $\mathrm{Cl}^{-}$channels and the pathogenesis of OA. 


\section{Extracellular Microenvironment and the Pathogenesis of OA}

Findings from previous studies have suggested that the osmotic pressure of joint fluid in healthy human joints is hypertonic at $404 \pm 57 \mathrm{mOsm} / \mathrm{L}$, while the osmotic pressure of joint fluid in joints with OA is reduced to $297 \pm 16.9$ $\mathrm{mOsm} / \mathrm{L} .{ }^{19-21}$ We know that changes in the osmotic pressure of the cell's environment will affect the volume of the cell and cause a series of changes in subsequent physiological and metabolic cell processes. ${ }^{22}$ Research by Yamada and other scholars has shown that, in healthy joints, articular cartilage cells are exposed to an overall relatively hypertonic dynamic osmotic environment and protect themselves from swelling and destruction through regulatory volume decrease (RVD). Furthermore, if the cell is in a relatively hypotonic environment for a long time and the pathological change in osmotic pressure exceeds the threshold of cell self-regulation, its RVD mechanism can no longer fully compensate, and the cell will continue to swell until death, triggering OA. ${ }^{23-25}$

In addition to the above-mentioned changes, the cellular edema resulting from the pathological hypotonic environment caused by OA can also lead to the activation of a volume-sensitive outward rectifier (VSOR) chlorine current. Relevant studies have confirmed that the VSOR chlorine current initiates RVD. Osteoarthritis can also cause acidification of the extracellular environment $(\mathrm{pH} \leq 5.0)$, which activates acid-sensitive outward rectification (ASOR) chloride currents. Under hypotonic and acidic stimulation, chondrocytes VSOR and ASOR chloride currents are co-activated. Prolonged activation will inactivate the VSOR current, impair the volume regulation ability of chondrocytes and aggravate chondrocyte death. ${ }^{26-29}$

ClC-3 is an ion channel encoded by the gene chloride voltage-gated channel 3 (CLCN3), which plays a key role in cell electrical activity and volume homeostasis, and is also involved in cell proliferation, migration, invasion and apoptosis. ClC-3 upregulates matrix metalloproteinase-9 (MMP-9), which promotes the pathological metabolism in extracellular matrix. ${ }^{30}$ Furthermore, under the long-term stimulation of a pathologically hypotonic environment (180 $\mathrm{mOsm} / \mathrm{L}$ ), ClC-3 participates in the regulation of the chloride current of chondrocytes through swelling-activated chloride current, cell swelling and RVD, which in turn causes the regulates prostaglandin $\mathrm{E}_{2}$ (PGE2) release in human OUMS-27 chondrocytes. ${ }^{23}$ Studies have confirmed that the expression of $\mathrm{ClC}-7$ channels in chondrocytes in a hypotonic state is reduced, and this change leads to hyperpolarization of the resting membrane potential of chondrocytes and increases cell death. Other researchers have actively downregulated the expression of ClC-7 channels in healthy chondrocytes, and similar changes can be observed in these cells. Thus, the ClC-7 channel and the aforementioned ClC-3 channel are both involved in the pathological changes of cartilage cell death and enhanced extracellular matrix catabolism in OA. ${ }^{31}$

Leucine-rich repeat-containing 8A (LRRC8A), which has been discovered recently, is proved to be the main component of the volume-regulated anion channel (VRAC). As an important component of the cell volume-sensitive chloride channel, LRRC8A participates in many pathophysiological processes. ${ }^{32-35}$ Studies have shown that artificially reducing the osmotic pressure of the extracellular fluid induces cell swelling, which activates the release of caspase-1 and the inflammatory factor IL-1 $\beta$ through the NLRP3 inflammasome, and then triggers a series of cellular inflammatory reactions, and a large number of activated inflammatory factors will eventually lead to inflammatory cell death. ${ }^{36,37}$ These pathophysiological processes are related to the abnormal increase in cell volume mediated by the abnormal activation of cell chloride ion channels. One previous study showed a functional deactivation and disordered distribution of LRRC8A chloride channels in OA chondrocytes, ${ }^{37}$ which we believe may be a new and valuable research direction.

\section{Abnormal Metabolism of the Extracellular Matrix and OA}

The metabolism of the articular cartilage matrix is the physiological basis for maintaining normal articular cartilage function. Cartilage matrix metabolism is divided into anabolism and catabolism, which co-exist in dynamic balance under normal circumstances. Chondrocytes secrete aggrecan, type II collagen, type I collagen, matrix metalloproteinases and other substances to maintain the homeostasis of the extracellular matrix, thereby maintaining normal structure and function of articular cartilage. ${ }^{6,38-40}$ Under the combined action of inflammatory factors, mechanical stimulation and other external inducing factors, the level of catabolism in the cartilage matrix will be significantly higher than the level of 
anabolism, resulting in imbalances in articular cartilage matrix metabolism, destruction of the extracellular matrix and the triggering of OA. ${ }^{41-44}$

Matrix metalloproteinase (MMP) is a large family of proteins that require metal ions such as $\mathrm{Ca}^{2+}$ and $\mathrm{Zn}^{2+}$ as cofactors for their active forms. Their main physiological function is to participate in the catabolism of the extracellular matrix. In the past, these proteins have mostly been associated with the migration, invasion or metastasis of tumor cells or other cells. Hsu et al have shown that proliferative vitreoretinopathy is related to increased catabolism of the extracellular matrix around retinal pigment epithelial cells. ${ }^{45}$ They found in further experiments that MMP-14 plays a key role in the degradation of the extracellular matrix, while the activity of MMP-14 is regulated by chloride intracellular channel 4 (CLIC4). ${ }^{45}$ However, when Guan et al studied the migration and invasion mechanisms of cancer cells, they found that ClC-3 can regulate the expression level of MMP-9 through the phosphorylation of the PI3K/Akt/ mTOR signaling pathway. In fact, they also found that cancer cells can express and secrete MMP-9 through this signal pathway to enhance the catabolism of extracellular matrix to achieve migration and invasion. ${ }^{46}$ Similarly, Wang et al showed that $\mathrm{ClC}-3$ can also increase the transcriptional activity of nuclear factor- $\mathrm{KB}(\mathrm{NF}-\mathrm{\kappa B})$ in cancer cells to upregulate the expression of MMP-3 and MMP-9, which can also promote malignant migration and invasion. ${ }^{47}$ In addition, in several reports on the pathogenesis of endometriosis, it was found that $\mathrm{ClC}-3$ can promote the abnormal metastasis and invasion of endometrial cells by mediating the upregulation of MMP-9. ${ }^{48-51}$

Previous studies have confirmed that excessive deposition of the extracellular matrix is an important cause of scar formation. The low expression of ClC-2 can reduce the expression of type I collagen, and type I collagen endangers the chondrocyte extracellular matrix. Excessive type I collagen deposition destroys the normal cartilage matrix structure, thereby causing increased degradation of the extracellular matrix, which initiates and accelerates the general manifestations of OA. ${ }^{50}$

Decreased estrogen levels are currently recognized as one of the risk factors for OA, which is most likely to appear in postmenopausal women. ${ }^{8,52-54}$ Studies have shown that in the absence of estrogen protection, the extracellular matrix of chondrocytes is prone to metabolic imbalance, and the catabolism is significantly increased. ${ }^{55-57}$ In addition, our team's previous research showed that normal estrogen levels significantly inhibit cell volume-activated chloride current and IL$1 \beta$ activated chloride current in healthy chondrocytes. Furthermore, estrogen inhibits IL-1 $\beta$-induced LRRC8A chloride channel distribution disorder through the microRNA-140 (miRNA-140) pathway. ${ }^{58}$ This indicates that the LRRC8A chloride channel may serve as an important target of estrogen and participate in the regulation of the occurrence and development of OA after menopause.

As mentioned earlier, in chondrocytes, enhanced extracellular matrix catabolism is also an important factor promoting the occurrence and progression of OA. Therefore, if this process also exists in cartilage, it means that chloride channels may participate in extracellular matrix metabolism by regulating the expression of the MMP protein family, thereby contributing to the occurrence and progression of OA. And chloride channels may be under the influence of changes of extracellular microenvironment, which initiate OA (Figure 1).

\section{Inflammatory Factors, Apoptosis and Pyroptosis in OA Inflammation Mediated by Chloride Channel in Chondrocyte Initiates Apoptosis and Pyroptosis}

Inflammation is an important protective response of the healthy human body from exogenous infection and injury, but inflammation often becomes the key point of disease progression in non-infectious diseases. Some previous studies have shown that inflammasomes play an important role in inflammatory response. ${ }^{59-61}$

Inflammasomes are an important part of the innate immune system including nod-like receptor pyrin domain 1 (NLRP1), NLRP3, NLRC4, AIM2 and Pyrin. And researches prove that NLRP3 is the one of most common inflammasome that regulates most of inflammation in cell and initiates abnormal cell death. NLRP3 inflammasome has been reported to participate in several chronic inflammatory diseases and some metabolic diseases. ${ }^{60}$ Furthermore, the mentioned studies showed that the activation of NLRP3 inflammasome depends on the ion-current, including $\mathrm{K}^{+}, \mathrm{Ca}^{2+}$ and $\mathrm{Cl}^{-}$, which is produced by the activation of cellular ion channel. ${ }^{62-64}$ 

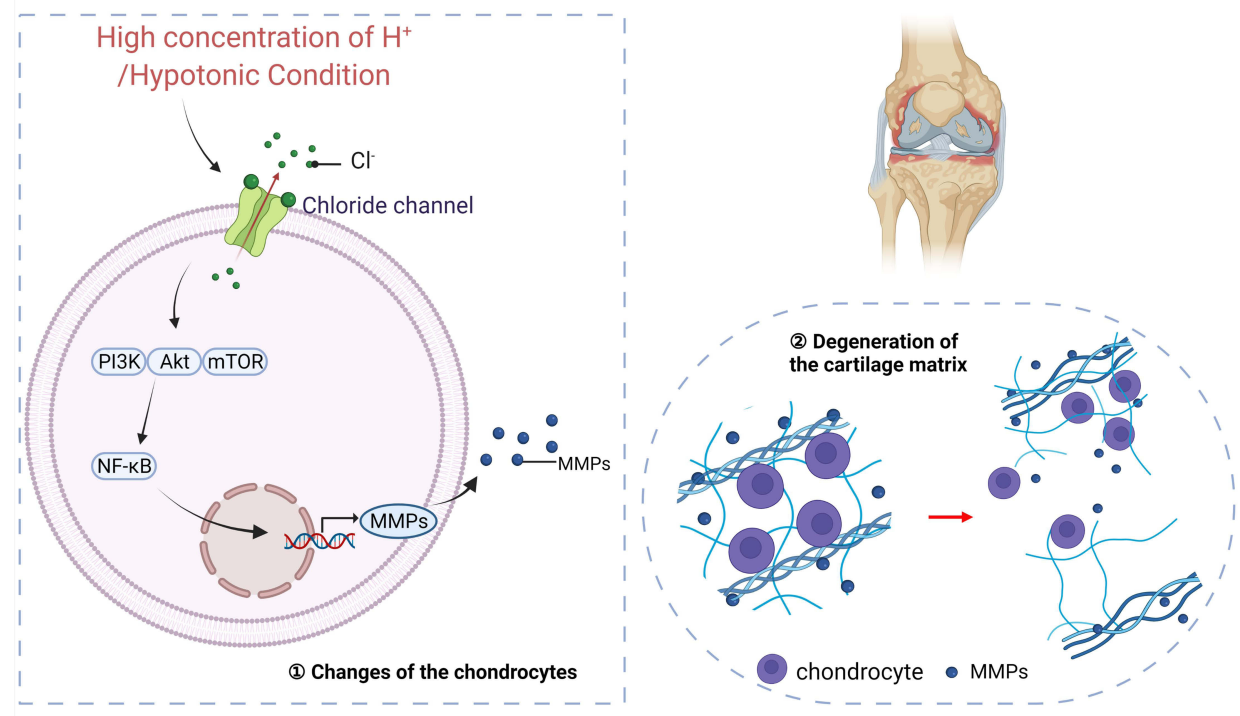

Figure I Abnormal extracellular microenvironment and extracellular matrix metabolism. In age-mediated degeneration of articular cartilage, presence of high concentration of proton and hypotonic microenvironment pathologically activates chloride channels on the chondrocyte membrane, which upregulates PI3K-Akt-mTOR signal pathway and activates NF- $\kappa B$ (1) Changes of the chondrocytes). Thus, the overexpressed MMPs which are upregulated by NF- $\kappa B$ induce abnormal metabolism of cartilage matrix and initiate OA (2) Degeneration of the cartilage matrix). Created with BioRender.com.

\section{Chloride Channels and NLRP3 Inflammasome in Inflammation}

The volume-sensitive chloride current of OA chondrocytes has been clinically shown to be significantly reduced, suggesting the RVD mechanism that depends on chloride channels is damaged. Our further study found that this phenomenon depends on inflammation-related proteins caspase-1, caspase-3, NLRP3 and high expression of the inflammation factor IL-1 $\beta$. Furthermore, IL-1 $\beta$ can induce the continuous opening of the chloride channel of healthy chondrocytes and damage the RVD mechanism of healthy chondrocytes, which participate in the pathogenesis of OA. ${ }^{37}$ The above-mentioned inflammation-related proteins cause abnormal intracellular skeleton distribution and abnormal extracellular matrix metabolism in healthy chondrocytes, thus damaging healthy cells or even leading to cell death. ${ }^{37,65}$ This further explains the mechanism of the aforementioned pathological processes in OA. Chen et al studied epithelial cells and macrophages to show that vitamin A affects the expression of downstream Chloride Channel Accessory 1 (CLCA1) and other $\mathrm{Ca}^{2+}$-sensitive chloride channels by regulating the expression of IL-4, which may be related to subsequent inflammation. ${ }^{66}$ However, there are no reports of experiments on chondrocytes with similar mechanisms. In addition, Kim et al found that the chloride current of the $\mathrm{Ca}^{2+}$-sensitive chloride channel was significantly enhanced in airway epithelial cells treated with IL-4, and they posited that this mechanism might be related to allergic rhinitis and other allergic reactions. ${ }^{67}$ These findings confirm the relationship between $\mathrm{Ca}^{2+}$-sensitive chloride channels and inflammatory factors in other areas of the body.

In the above-mentioned description, extracellular hypotonicity induces activation of chloride channels and initiates RVD in cell. Green et al found that extracellular hypotonic pressure induces the activation of NLRP3 inflammasomes, which convert the inactive precursors of IL-1 $\beta$ and IL-18 into their active forms and activate the downstream volumesensitive chloride channel of LRRC8A. ${ }^{68}$ This process is considered one of the mechanisms of cell inflammatory necrosis. ${ }^{59,61,68}$ Furthermore, other research demonstrated that this procedure also responds to other stimulations. ${ }^{69}$ And recent studies showed that inhibition of chloride channels blocks the release of IL-1 $\beta$ and activation of NLRP3 inflammasome, which is regarded as a potential target for advanced treatment or medication. ${ }^{36}$

In addition to those located on the membrane, CLIC family is an important part of chloride channel proteins. It is composed of six highly conserved subtypes, which can exist and function either as soluble monomers or as a wholemembrane channel after structural rearrangement. They are widely involved in the inflammatory response of tissue damage and the progression of tumors. CLICs are observed to play a similar function on cell membrane as the traditional 
transmembrane VRAC, although they were regarded as the intracellular channel which transport $\mathrm{Cl}^{-}$only inside the cell. ${ }^{60}$ Related research has shown that CLIC-1 is highly expressed on the tumor cells of a variety of human cancers, and it is involved in tumor progression and metastasis. ${ }^{70}$ Studies have shown that CLIC-1 can shuttle between the cytoplasm and cell membrane, and participate in the process of regulating cell adhesion to the extracellular matrix. This process is achieved by CLIC-1 regulating the new extracellular matrix through the PIP5Ks signaling pathway. ${ }^{70}$ TME16A and CLCA1 are $\mathrm{Ca}^{2+}$-activated chloride channels that have been discovered recently. ${ }^{71,72}$ Previous studies have shown that they participate in the enhancement of the production and secretion of inflammation mucus in the airway, which is regulated by high levels of IL-13. This process is closely related to the production of inflammatory mucus, ${ }^{72,73}$ although no similar studies of chondrocytes exist.

Classic apoptosis is a process of caspase-dependent programmed cell death, which is beneficial to organisms generally. However, pyroptosis is defined as another kind of caspase-dependent programmed cell death, which is initiated by inflammasomes and other inflammatory factors. Therefore, the early studies of pyroptosis focused on pathophysiological processes of infectious inflammation and researches did discover and demonstrate significant results. ${ }^{74}$ Recently, a myriad of researches have demonstrated that pyroptosis is also involved in and regulates aseptic inflammation through some specific pathways. ${ }^{75}$ Further studies showed that NLRP3 inflammasome mediates pyroptosis in articular cartilage, which initiates chondrocyte death and cartilage degradation. ${ }^{76} \mathrm{~A}$ case in point, chronic hyperplasia of inflammatory synovium also initiates cartilage degradation, and recent study proofed that IL-1 $\beta$ may be produced from inflammatory synovium. ${ }^{77}$ And Zhao et al demonstrated that NLRP3 inflammasome interacts with fibroblast-like synoviocyte to regulate the expression and activation of IL-1ßand IL-18, which were proved to cause the pyroptosis of chondrocyte and initiate cartilage degradation. ${ }^{78}$ And Domingo et al demonstrated that CLIC plays a critical function in inflammation mediated by NLRP3 inflammasome and the activation of CLIC1 and CLIC4 activates NLRP3 inflammasome and induces pyroptosis. $^{79}$ Moreover, CLIC-dependent chloride current regulated downstream potassium efflux and mitochondrial reactive oxygen species (ROS), which actually activates NLRP3 inflammasome. Their research demonstrated that CLICdependent chloride efflux is an essential and proximal upstream event for NLRP3 inflammasome activation. ${ }^{60}$ Zhao et al demonstrated that extracellular lactate accumulation promotes activation of NLRP3 inflammasome and degeneration of extracellular matrix, which initiate inflammation and pyroptosis ${ }^{80}$ (Figure 2). Pyroptosis not only occurs in chondrocyte and cause cartilage degradation but also occurs in other tissues. Synovial macrophage can provide a protective barrier for the articular cartilage and restricts the severe inflammatory reaction. ${ }^{81}$ However, some researchers observed that pyroptosis caused by overexpression of inflammasomes and inflammatory factors occurs in synovial macrophage in animal model with knee OA. They believed this phenomenon causes reduction of synovial macrophage and initiates uncontrolled synovial inflammatory reaction in joint. ${ }^{75}$ In addition, some specific kinds of microRNA were proved to inhibit chondrocyte pyroptosis and delay progression of OA. ${ }^{82-84}$

We realized that pyroptosis may not only destroy imperative cell in joint but also destroy the barrier. And perhaps we can attempt to develop and design some medications to inhibit pyroptosis in joint with OA. Fortunately, advanced researches demonstrated that some medication or biological active substance including hypertonicity, hydrogen sulfide $\left(\mathrm{H}_{2} \mathrm{~S}\right)$ donor, dihydrotanshinone I and berberine could inhibit inflammation and pyroptosis by regulating activation of NLRP3 inflammasome and other factors. ${ }^{36,85-87}$ And other researchers have designed some medication to restrict chondrocyte pyroptosis through regulating NLRP3 inflammatory in vitro, including icariin, indomethacin and loganin. ${ }^{88-90}$

Some scholars have confirmed the above-mentioned pathophysiological process by means of animal experiments, which have shown that the abnormal pathological changes in the chondrocyte volume in a hypotonic environment may be related to the increased activity of caspase- 3 or caspase- $7 .{ }^{91}$ This suggests that the final outcome of the process is related to apoptosis. In their research on tumor cells, Zhou et al found that ClC-3 can be induced by dihydroartemisinin (DHA) to upregulate its expression and ClC-3 leads to efflux of chloride ions and apoptotic volume decrease (AVD), which is similar to RVD. Furthermore, the above-mentioned process upregulates the expression of caspase-3, which induces apoptosis of cancer cells. ${ }^{92}$ Studies on other tissues or cells in the intestines and blood vessels have found similar $\mathrm{ClC}$ family proteins involved in the process of caspase family-induced apoptosis. ${ }^{93}$ 


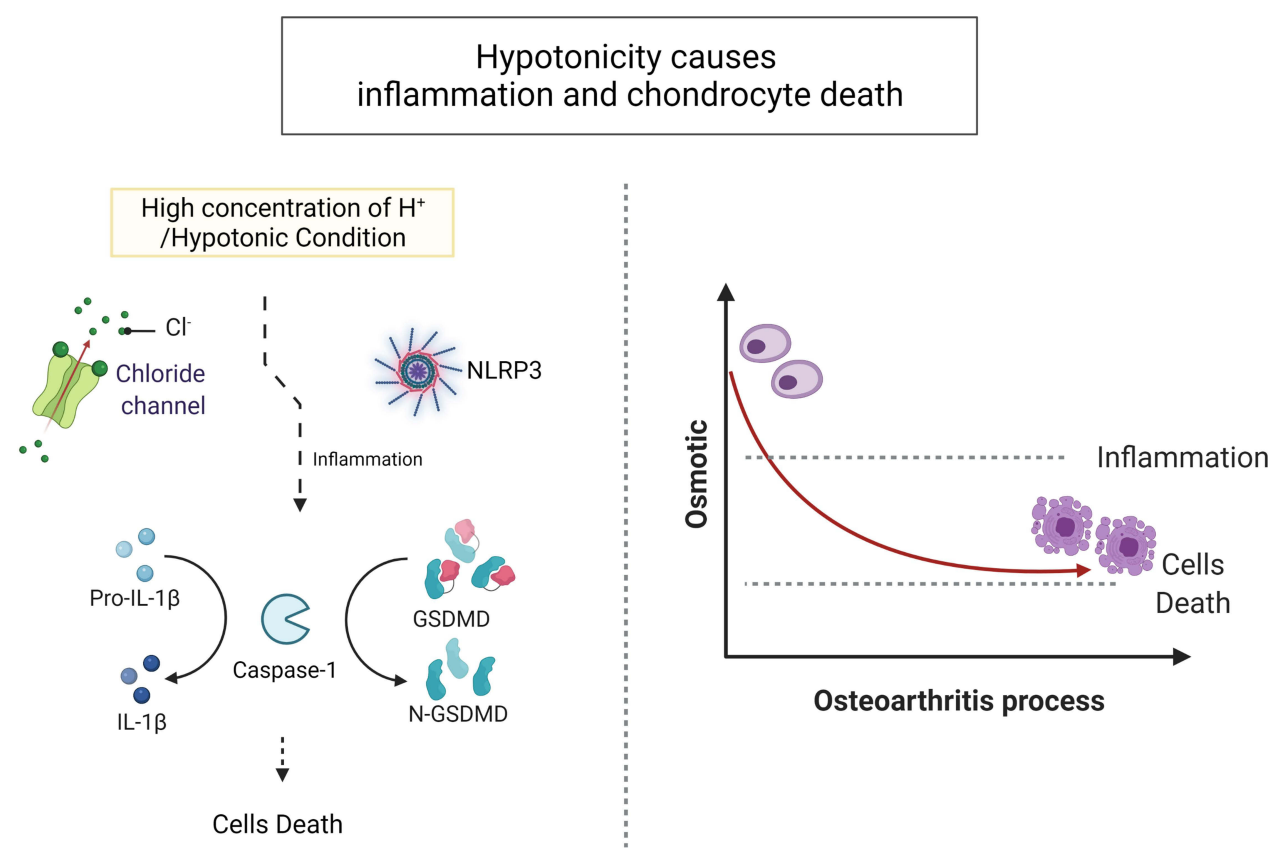

Figure 2 Hypotonicity causes inflammation and chondrocyte death. Presence of high concentration of proton and hypotonic microenvironment pathologically activates chloride channels on the chondrocyte membrane and enhances transmembrane $\mathrm{Cl}^{-}$current. Enhanced $\mathrm{Cl}^{-}$current initiates inflammation through NLRP3 inflammasome, which activates IL-I $\beta$ and N-GSDMD. These typical inflammatory factors cause pathological inflammation in articular cartilage and causes abnormal cell death (apoptosis and pyroptosis). Created with BioRender.com.

The mentioned studies examined the $\mathrm{ClC}-3$ and $\mathrm{ClC}-7$ subtypes of the $\mathrm{ClC}$ chloride channel family. Numerous studies have confirmed that these subtypes can participate in the regulation of the apoptosis process induced by the caspase protein family. ${ }^{31,92,94}$ Other studies have shown that overexpression of ClC-2 can reduce the expression of caspase-3 and caspase-9, and thereby inhibit the occurrence of cell apoptosis. ${ }^{95}$ Previous studies have confirmed the presence of ClC-3 mRNA expression in articular cartilage. ${ }^{96}$ However, there have been few studies across the spectrum of ClC chloride channel proteins on the mechanism of apoptosis induced by caspase family proteins in human chondrocytes. We believe elucidation of this phenomenon may direct future novel research.

\section{Mechanical Interaction and OA}

Mechanical stimulation usually causes changes in cell volume. Previous studies demonstrated that abnormal mechanical force, as an important factor, leads to cartilaginous lesion clinically. ${ }^{8,97}$ Overload of joints under physiological or pathological conditions will significantly affect the normal metabolism of cartilage cells. For example, a high internal dynamic load of joints and abnormal gait movement will cause an abnormal distribution of load forces in joints. ${ }^{98-100}$ Furthermore, mechanical factors such as anterior cruciate ligament injury or rupture and incomplete meniscus will also affect the distribution of the entire joint load. ${ }^{101}$ These mechanical factors are considered an important cause of OA. However, how this change affects cells under normal physiological conditions is still inconclusive, and this topic is the focus of recent research on OA pathology. Wang et al found that in the MC3T3-E1 cell line, mechanical stimulation induces the upregulation of $\mathrm{ClC}-3$ expression. In addition, increased mechanical stimulation and $\mathrm{ClC}-3$ overexpression can also stimulate the expression of osteogenic markers, such as alkaline phosphatase (ALP), bone sialoprotein (BSP), and osteocalcin (Oc). Furthermore, the authors pointed out that RUNX family transcription factor 2 (Runx2) and transforming growth factor-1 $\alpha$ (TGF-1 $\alpha$ ) may also be involved in this regulation ${ }^{102}$ (Figure 3). The above-mentioned process will eventually lead to osteoblast differentiation. Under normal circumstances, fracture repair may depend on this signaling pathway, but in patients with OA, this may also be one of the mechanisms of subchondral bone sclerosis. The unique cellular physiological functions of some kinds of cells depend on the transport of chloride ions between the extracellular and intracellular environments. Specifically, Harasztosi and Gummer discovered a chlorine-sensitive protein 


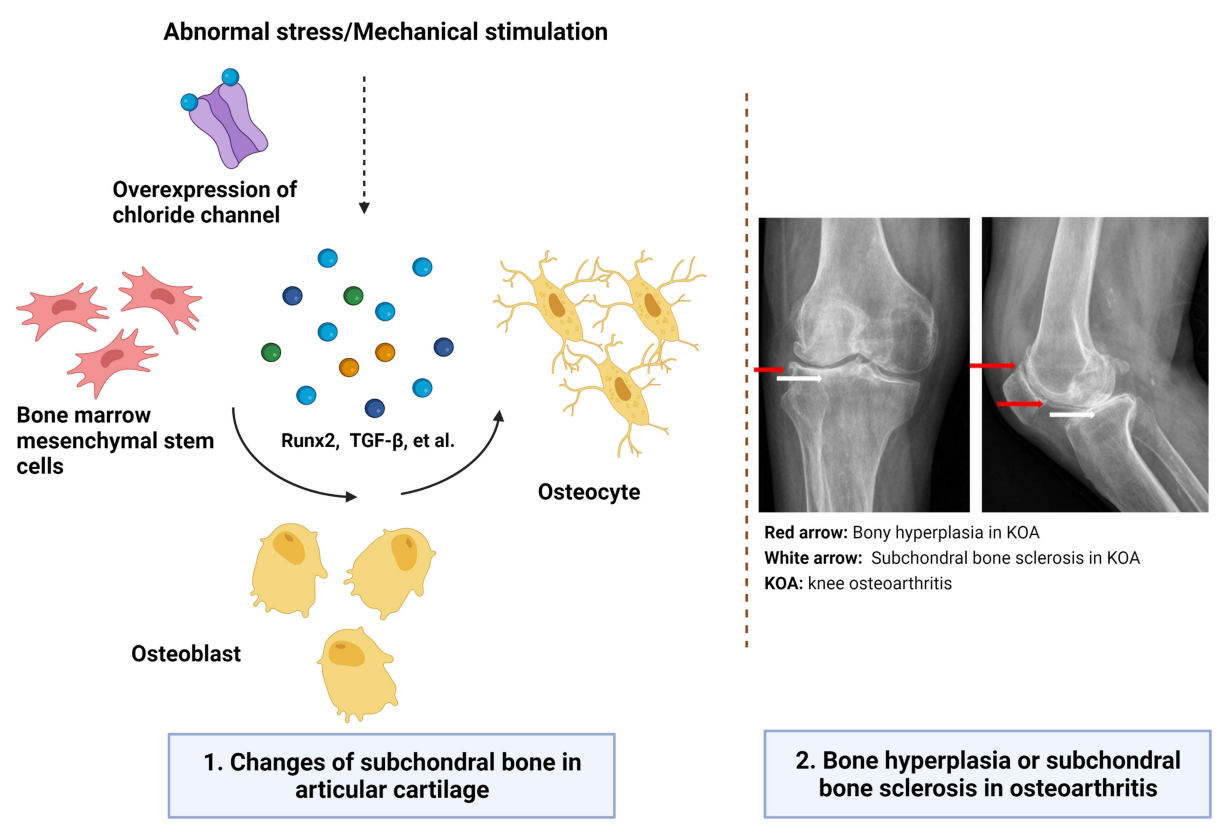

Figure 3 Mechanical interaction causes osteoarthritis. Abnormal stress and long-term mechanical stimulation upregulate the expression level of chloride channels, which induce the overexpression of osteogenic factors (Runx2, TGF- $\beta$, etc). These biomarkers stimulate the differentiation from bone marrow mesenchymal cells (BMSCs) to osteoblasts, and finally differentiate to osteocyte. (I. Changes of subchondral bone in articular cartilage) Therefore, the mechanism explains bone hyperplasia or subchondral bone sclerosis in OA. (2. Bone hyperplasia or subchondral bone sclerosis in osteoarthritis). Created with BioRender.com.

Abbreviation: KOA, knee osteoarthritis.

called prestin in cochlea, and the skeletal protein of the experimental cell was deconstructed after treatment with 9-anthracenecarboxylic acid (9-AC), a chloride channel inhibitor that inhibits prestin function. ${ }^{103}$ These results confirm the importance of prestin, a chlorine-sensitive protein, in the normal function of the cochlea. We believe that a similar mechanism may also exist in chondrocytes, whereby the activity of chloride channel proteins is inhibited, causing the deconstruction of the structural proteins in chondrocytes to trigger cell death and OA. As mentioned above, highintensity exercise and inappropriate sports are believed to be a risk factor of cartilage degradation and OA. However, a recent study demonstrated that moderate-intensity exercise alleviates chondrocyte pyroptosis by promoting autophagy of chondrocyte, which avoids the release of inflammasomes. ${ }^{104}$

\section{Conclusion}

$\mathrm{OA}$ is a common disease worldwide, mainly manifested in pathophysiological processes such as cartilage degeneration, local aseptic inflammation and bone hyperplasia. However, the mechanisms of occurrence and progression of these pathophysiological processes are not conclusive. We know that cartilage tissue is an organic whole composed of chondrocytes, the extracellular matrix and the microenvironment. Once the factors that maintain homeostasis appear pathologically, they are very likely to initiate or trigger OA through a certain pathway. Many recent studies have focused on the cellular chloride ion channel, inflammatory factors and aseptic inflammation, which involved in the pathogenesis of OA. Moreover, these factors also participate in pathological processes including change of extracellular microenvironment, break of balance in extracellular matrix metabolism, apoptosis and pyroptosis. In recent years, more and more studies have also shown that chloride channels are widely present in various types of cells and play a role in regulating various physiological and pathological activities through different pathways and mechanisms. Moreover, more and more studies have also demonstrated that inflammatory factors and inflammation regulated by chloride channels participate to the regulation of OA pathogenesis. However, most of the above-mentioned studies are based on other types of cells. We believe that future studies must use the above-mentioned conclusions as the starting point, for research on chondrocytes, and a better understanding of the mechanism of OA will provide a theoretical basis for potential new medication targets in the future. Fortunately, some researchers have gained achievements in novel medication, which targets key factors 
involved in the above-mentioned pathological processes (including chloride channels, NLRP3 inflammasome, etc). Furthermore, more novel and potential medications are supposed to be developed and designed to ameliorate OA through regulating $\mathrm{pH}$ and osmotic pressure in synovial fluid, controlling inflammation and inhibiting apoptosis and pyroptosis of cells. We believe that this may be a potential therapeutic strategy for OA.

\section{Acknowledgments}

The authors thank LetPub (www.letpub.com) for its linguistic assistance during the preparation of this manuscript.

\section{Funding}

This study was supported by the National Natural Science Foundation of China $(81800785,81972085,81902303)$, the Natural Science Foundation of Guangdong Province (2018A0303100027, 2020A151501048, 2021A1515010706), Guangdong Provincial Key Clinical Discipline-Orthopedics (2000005), the Sanming Project of Shenzhen Health and Family Planning Commission (SZSM201612086), Shenzhen Science and Technology Planning (JCYJ20180228163401333, JCYJ20190806170612680, JCYJ20190806164216661, RCBS20200714114856299), the Shenzhen Key Medical Discipline Construction Fund (SZXK025), the discipline construction Capacity Improvement project of Shenzhen Municipal Health Commission (SZXJ2018065), and Clinical Research Project of Shenzhen Second People's Hospital (20203357028).

\section{Disclosure}

The authors report no conflicts of interest in this work.

\section{References}

1. Barrett-Jolley R, Lewis R, Fallman R, et al. The emerging chondrocyte channelome. Front Physiol. 2010;1:135. doi:10.3389/fphys.2010.00135

2. Oláh T, Cai X, Michaelis JC, et al. Comparative anatomy and morphology of the knee in translational models for articular cartilage disorders. Part I: large animals. Ann Anat. 2021;235:151680.

3. Yamamura H, Suzuki Y, Imaizumi Y. Physiological and pathological functions of $\mathrm{Cl}$ channels in chondrocytes. Biol Pharm Bull. 2018;41 (8):1145-1151. doi:10.1248/bpb.b18-00152

4. Hodgkinson T, Kelly DC, Curtin CM, et al. Mechanosignalling in cartilage: an emerging target for the treatment of osteoarthritis. Nat Rev Rheumatology. 2021;3:1-18.

5. Gilbert SJ, Bonnet CS, Blain EJ. Mechanical cues: bidirectional reciprocity in the extracellular matrix drives mechano-signalling in articular cartilage. Int J Mol Sci. 2021;22(24):13595. doi:10.3390/ijms222413595

6. Liu Y, Shah KM, Luo J. Strategies for articular cartilage repair and regeneration. Front Bioeng Biotechnol. 2021;9:770655. doi:10.3389/ fbioe. 2021.770655

7. Deng J, Zong Z, Su Z, et al. Recent advances in pharmacological intervention of osteoarthritis: a biological aspect. Front Pharmacol. $2021 ; 12$. doi:10.3389/fphar.2021.772678

8. Hunter DJ, Bierma-Zeinstra S. Osteoarthritis. Lancet. 2019;393(10182):1745-1759. doi:10.1016/S0140-6736(19)30417-9

9. Hunter DJ, Schofield D, Callander E. The individual and socioeconomic impact of osteoarthritis. Nat Rev Rheumatol. 2014;10(7):437-441. doi:10.1038/nrrheum.2014.44

10. Vos T, Flaxman AD, Naghavi M, et al. Years lived with disability (YLDs) for 1160 sequelae of 289 diseases and injuries 1990-2010: a systematic analysis for the Global Burden of Disease Study 2010. Lancet. 2012;380(9859):2163-2196.

11. Garcia G, Martínez-Rojas VA, Rocha-González HI, et al. Evidence for the participation of $\mathrm{Ca}(2+)$-activated chloride channels in formalin-induced acute and chronic nociception. Brain Res. 2014;1579:35-44. doi:10.1016/j.brainres.2014.07.011

12. Ebbinghaus M, Gajda M, Holtzman MJ, et al. Does chloride channel accessory 3 have a role in arthritis pain? A study on murine antigen-induced arthritis. Neurosci Lett. 2014;576:40-44. doi:10.1016/j.neulet.2014.05.051

13. Jentsch T, Stein V, Weinreich F, et al. Molecular structure and physiological function of chloride channels. Physiol Rev. 2002;82(2):503-568. doi:10.1152/physrev.00029.2001

14. Smith KR, Kittler JT. The cell biology of synaptic inhibition in health and disease. Curr Opin Neurobiol. 2010;20(5):550-556. doi:10.1016/j. conb.2010.06.001

15. Mohler H, Malherbe P, Draguhn A, et al. GABAA-receptors: structural requirements and sites of gene expression in mammalian brain. Neurochem Res. 1990;15(2):199-207. doi:10.1007/BF00972210

16. Zhang H, Deng Z, Yang L, et al. The AQP-3 water channel is a pivotal modulator of glycerol-induced chloride channel activation in nasopharyngeal carcinoma cells. Int J Biochem Cell Biol. 2016;72:89-99. doi:10.1016/j.biocel.2016.01.009

17. Deng Z, Li W, Xu J, et al. ClC-3 chloride channels are involved in estradiol regulation of bone formation by MC3T3-E1 osteoblasts. $J$ Cell Biochem. 2018;120:8366-8375.

18. Deng Z, Peng S, Zheng Y, et al. Estradiol activates chloride channels via estrogen receptor- $\alpha$ in the cell membranes of osteoblasts. Am J Physiol Cell Physiol. 2017;313(2):C162-c172. doi:10.1152/ajpcell.00014.2017 
19. Bertram KL, Krawetz RJ. Osmolarity regulates chondrogenic differentiation potential of synovial fluid derived mesenchymal progenitor cells. Biochem Biophys Res Commun. 2012;422(3):455-461. doi:10.1016/j.bbrc.2012.05.015

20. Nguyen TD, Oloyede A, Singh S, et al. Investigation of the effects of extracellular osmotic pressure on morphology and mechanical properties of individual chondrocyte. Cell Biochem Biophys. 2016;74(2):229-240. doi:10.1007/s12013-016-0721-1

21. Johnson ZI, Shapiro IM, Risbud MV. Extracellular osmolarity regulates matrix homeostasis in the intervertebral disc and articular cartilage: evolving role of TonEBP. Matrix Biol. 2014;40:10-16. doi:10.1016/j.matbio.2014.08.014

22. Wang M, Yang Y, Han L, et al. Cell mechanical microenvironment for cell volume regulation. J Cell Physiol. 2020;235(5):4070-4081. doi:10.1002/jcp.29341

23. Yamada S, Suzuki Y, Bernotiene E, et al. Swelling-activated ClC-3 activity regulates prostaglandin E release in human OUMS-27 chondrocytes. Biochem Biophys Res Commun. 2021;537:29-35. doi:10.1016/j.bbrc.2020.12.068

24. Moretti J, Blander JM. Increasing complexity of NLRP3 inflammasome regulation. J Leukoc Biol. 2021;109(3):561-571.

25. Wang Z, Irianto J, Kazun S, et al. The rate of hypo-osmotic challenge influences regulatory volume decrease (RVD) and mechanical properties of articular chondrocytes. Osteoarthr Cartil. 2015;23(2):289-299. doi:10.1016/j.joca.2014.11.003

26. Komori T. Cell death in chondrocytes, osteoblasts, and osteocytes. Int J Mol Sci. 2016;17(12):2045. doi:10.3390/ijms17122045

27. Kittl M, Winklmayr M, Helm K, et al. Acid- and volume-sensitive chloride currents in human chondrocytes. Front Cell Dev Biol. 2020;8:583131. doi:10.3389/fcell.2020.583131

28. Hall A. The role of chondrocyte morphology and volume in controlling phenotype-implications for osteoarthritis, cartilage repair, and cartilage engineering. Curr Rheumatol Rep. 2019;21(8):38. doi:10.1007/s11926-019-0837-6

29. Ponce A, Jimenez-Peña L, Tejeda-Guzman C. The role of swelling-activated chloride currents (I(CL, swell)) in the regulatory volume decrease response of freshly dissociated rat articular chondrocytes. Cell Physiol Biochem. 2012;30(5):1254-1270.

30. Riemma G, Laganà AS, Schiattarella A, et al. Ion channels in the pathogenesis of endometriosis: a cutting-edge point of view. Int J Mol Sci. 2020;21(3):1114. doi:10.3390/ijms21031114

31. Kurita T, Yamamura H, Suzuki Y, et al. The ClC-7 chloride channel is downregulated by hypoosmotic stress in human chondrocytes. Mol Pharmacol. 2015;88(1):113-120. doi:10.1124/mol.115.098160

32. Voss FK, Ullrich F, Münch J, et al. Identification of LRRC8 heteromers as an essential component of the volume-regulated anion channel VRAC. Science. 2014;344(6184):634-638. doi:10.1126/science.1252826

33. Chen L, König B, Liu T, et al. More than just a pressure relief valve: physiological roles of volume-regulated LRRC8 anion channels. Biol Chem. 2019;400(11):1481-1496.

34. Liu T, Stauber T. The volume-regulated anion channel LRRC8/VRAC is dispensable for cell proliferation and migration. Int J Mol Sci. 2019;20 (11):453.

35. Zhang H, Deng Z, Zhang D, et al. High expression of leucine-rich repeat-containing $8 \mathrm{~A}$ is indicative of a worse outcome of colon cancer patients by enhancing cancer cell growth and metastasis. Oncol Rep. 2018;40(3):1275-1286.

36. Compan V, Baroja-Mazo A, López-Castejón G, et al. Cell volume regulation modulates NLRP3 inflammasome activation. Immunity. 2012;37 (3):487-500. doi:10.1016/j.immuni.2012.06.013

37. Deng Z, Lin Z, Zhong Q, et al. Interleukin 1 beta-induced chloride currents are important in osteoarthritis onset: an in vitro study. Acta Biochim Biophys Sin (Shanghai). 2021;53(4):400-409. doi:10.1093/abbs/gmab010

38. Vaca-González J, Guevara JM, Moncayo MA, et al. Biophysical stimuli: a review of electrical and mechanical stimulation in hyaline cartilage. Cartilage. 2019;10(2):157-172. doi:10.1177/1947603517730637

39. Nguyen TH, Duong CM, Nguyen X-H, et al. Mesenchymal stem cell-derived extracellular vesicles for osteoarthritis treatment: extracellular matrix protection, chondrocyte and osteocyte physiology, pain and inflammation management. Cells. 2021;10(11):2887. doi:10.3390/ cells 10112887

40. Park S, Bello A, Arai Y, et al. Functional duality of chondrocyte hypertrophy and biomedical application trends in osteoarthritis. Pharmaceutics. 2021;13(8):1139. doi:10.3390/pharmaceutics13081139

41. Zhang H, Wei Q-F, Wang S-J, et al. LncRNA HOTAIR alleviates rheumatoid arthritis by targeting miR-138 and inactivating NF- $\mathrm{kB}$ pathway. Int Immunopharmacol. 2017;50:283-290. doi:10.1016/j.intimp.2017.06.021

42. Xie Q, Wei M, Kang X, et al. Reciprocal inhibition between miR-26a and NF- $\mathrm{kB}$ regulates obesity-related chronic inflammation in chondrocytes. Biosci Rep. 2015;35(3). doi:10.1042/BSR20150071

43. Duan L, Liang Y, Ma B, et al. Epigenetic regulation in chondrocyte phenotype maintenance for cell-based cartilage repair. Am J Transl Res. 2015;7(11):2127-2140.

44. Duan L, Ma B, Liang Y, et al. Cytokine networking of chondrocyte dedifferentiation in vitro and its implications for cell-based cartilage therapy. Am J Transl Res. 2015;7(2):194-208.

45. Hsu KS, Otsu W, Li Y, et al. CLIC4 regulates late endosomal trafficking and matrix degradation activity of MMP14 at focal adhesions in RPE cells. Sci Rep. 2019;9(1):12247. doi:10.1038/s41598-019-48438-0

46. Guan Y, Luan Y, Xie Y, et al. Chloride channel-3 is required for efficient tumour cell migration and invasion in human cervical squamous cell carcinoma. Gynecol Oncol. 2019;153(3):661-669. doi:10.1016/j.ygyno.2019.03.006

47. Wang B, Xie J, He H-Y, et al. Suppression of CLC-3 chloride channel reduces the aggressiveness of glioma through inhibiting nuclear factor- $\mathrm{kB}$ pathway. Oncotarget. 2017;8(38):63788-63798. doi:10.18632/oncotarget.19093

48. Chung H, Wen Y, Chun S-H, et al. Matrix metalloproteinase-9 and tissue inhibitor of metalloproteinase-3 mRNA expression in ectopic and eutopic endometrium in women with endometriosis: a rationale for endometriotic invasiveness. Fertil Steril. 2001;75(1):152-159. doi:10.1016/ S0015-0282(00)01670-8

49. Xin L, Hou Q, Xiong QI, et al. Association between matrix metalloproteinase-2 and matrix metalloproteinase-9 polymorphisms and endometriosis: a systematic review and meta-analysis. Biomed Rep. 2015;3(4):559-565. doi:10.3892/br.2015.447

50. Sun L, Dong Y, Zhao J, et al. The CLC-2 chloride channel modulates ECM synthesis, differentiation, and migration of human conjunctival fibroblasts via the PI3K/Akt signaling pathway. Int J Mol Sci. 2016;17(6):910. doi:10.3390/ijms17060910

51. Bostanci Durmus A, Dincer Cengiz S, Yılmaz H, et al. The levels of matrix metalloproteinase-9 and neutrophil gelatinase-associated lipocalin in different stages of endometriosis. J Obstet Gynaecol. 2019;39(7):991-995. doi:10.1080/01443615.2019.1584889 
52. Chaisson C, Zhang Y, McAlindon TE, et al. Radiographic hand osteoarthritis: incidence, patterns, and influence of pre-existing disease in a population based sample. J Rheumatol. 1997;24(7):1337-1343.

53. Xiao YP, Tian F-M, Dai M-W, et al. Are estrogen-related drugs new alternatives for the management of osteoarthritis? Arthritis Res Ther. 2016;18:151. doi:10.1186/s13075-016-1045-7

54. Wilson MG, Michet CJ, Ilstrup DM, et al. Idiopathic symptomatic osteoarthritis of the hip and knee: a population-based incidence study. Mayo Clin Proc. 1990;65(9):1214-1221. doi:10.1016/S0025-6196(12)62745-1

55. Liu H, Yang S-D, Xu Y, et al. Protective role of $17 \beta$-estradiol on tumor necrosis factor- $\alpha$-induced apoptosis in human nucleus pulposus cells. Mol Med Rep. 2017;16(2):1093-1100. doi:10.3892/mmr.2017.6690

56. Wang Y, Liu Z, Wang Q, et al. Early detection of tibial cartilage degradation and cancellous bone loss in an ovariectomized rat model. Biomed Res Int. 2017;2017:9654056.

57. Hu Q, Ecker M. Overview of MMP-13 as a promising target for the treatment of osteoarthritis. Int J Mol Sci. 2021;22(4):e12.

58. Liang Y, Duan L, Xiong J, et al. E2 regulates MMP-13 via targeting miR-140 in IL-1ß-induced extracellular matrix degradation in human chondrocytes. Arthritis Res Ther. 2016;18(1):105. doi:10.1186/s13075-016-0997-y

59. He Y, Hara H, Nunez G. Mechanism and regulation of NLRP3 inflammasome activation. Trends Biochem Sci. 2016;41(12):1012-1021.

60. Tang T, Lang X, Xu C, et al. CLICs-dependent chloride efflux is an essential and proximal upstream event for NLRP3 inflammasome activation. Nat Commun. 2017;8(1):202. doi:10.1038/s41467-017-00227-x

61. Cullen SP, Kearney C, Clancy D, et al. Diverse activators of the NLRP3 inflammasome promote IL-1beta secretion by triggering necrosis. Cell Rep. 2015;11(10):1535-1548. doi:10.1016/j.celrep.2015.05.003

62. Lee GS, Subramanian N, Kim AI, et al. The calcium-sensing receptor regulates the NLRP3 inflammasome through Ca2+ and cAMP. Nature. 2012;492(7427):123-127. doi:10.1038/nature11588

63. Munoz-Planillo R, Kuffa P. K(+) efflux is the common trigger of NLRP3 inflammasome activation by bacterial toxins and particulate matter. Immunity. 2013;38(6):1142-1153. doi:10.1016/j.immuni.2013.05.016

64. Daniels MJ, Rivers-Auty J, Schilling T, et al. Fenamate NSAIDs inhibit the NLRP3 inflammasome and protect against Alzheimer's disease in rodent models. Nat Commun. 2016;7:12504. doi:10.1038/ncomms12504

65. Son YO, Park S, Kwak J-S, et al. Estrogen-related receptor gamma causes osteoarthritis by upregulating extracellular matrix-degrading enzymes. Nat Commun. 2017;8(1):2133. doi:10.1038/s41467-017-01868-8

66. Chen C, Smith AD, Cheung L, et al. Potentiation of IL-4 signaling by retinoic acid in intestinal epithelial cells and macrophages-mechanisms and targets. Front Immunol. 2020;11:605. doi:10.3389/fimmu.2020.00605

67. Kim HJ, Nam YR, Nam JH. Flos magnoliae inhibits chloride secretion via ANO1 inhibition in Calu-3 cells. Am J Chin Med. 2018;46 (5):1079-1092. doi:10.1142/S0192415X18500568

68. Green JP, Swanton T, Morris LV, et al. LRRC8A is essential for hypotonicity-, but not for DAMP-induced NLRP3 inflammasome activation. Elife. 2020;9. doi:10.7554/eLife.59704

69. Osei-Owusu J, Yang J, del Carmen Vitery M, et al. Molecular biology and physiology of volume-regulated anion channel (VRAC). Curr Top Membr. 2018;81:177-203.

70. Peng JM, Lin S-H, Yu M-C, et al. CLIC1 recruits PIP5K1A/C to induce cell-matrix adhesions for tumor metastasis. J Clin Invest. 2021;131(1) doi:10.1172/JCI133525

71. Cabrita I, Benedetto R, Wanitchakool P, et al. TMEM16A mediates mucus production in human airway epithelial cells. Am J Respir Cell Mol Biol. 2021;64(1):50-58.

72. Mertens TC, Hiemstra PS, Taube C. Azithromycin differentially affects the IL-13-induced expression profile in human bronchial epithelial cells. Pulm Pharmacol Ther. 2016;39:14-20. doi:10.1016/j.pupt.2016.05.005

73. Qin Y, Jiang Y, Sheikh AS, et al. Interleukin-13 stimulates MUC5AC expression via a STAT6-TMEM16A-ERK1/2 pathway in human airway epithelial cells. Int Immunopharmacol. 2016;40:106-114. doi:10.1016/j.intimp.2016.08.033

74. Bergsbaken T, Fink SL, Cookson BT. Pyroptosis: host cell death and inflammation. Nat Rev Microbiol. 2009;7(2):99-109. doi:10.1038/ nrmicro2070

75. Zhang L, Xing R, Huang Z, et al. Inhibition of synovial macrophage pyroptosis alleviates synovitis and fibrosis in knee osteoarthritis. Mediators Inflamm. 2019;2019:2165918. doi:10.1155/2019/2165918

76. An S, Hu H, Li Y, et al. Pyroptosis plays a role in osteoarthritis. Aging Dis. 2020;11(5):1146-1157. doi:10.14336/AD.2019.1127

77. Borgonio Cuadra VM, González-Huerta NC, Romero-Córdoba S, et al. Altered expression of circulating microRNA in plasma of patients with primary osteoarthritis and in silico analysis of their pathways. PLoS One. 2014;9(6):e97690. doi:10.1371/journal.pone.0097690

78. Zhao LR, Xing RL, Wang PM, et al. NLRP1 and NLRP3 inflammasomes mediate LPS/ATPinduced pyroptosis in knee osteoarthritis. Mol Med Rep. 2018;17(4):5463-5469.

79. Domingo-Fernandez R, Coll RC, Kearney J, et al. The intracellular chloride channel proteins CLIC1 and CLIC4 induce IL-1beta transcription and activate the NLRP3 inflammasome. J Biol Chem. 2017;292(29):12077-12087. doi:10.1074/jbc.M117.797126

80. Zhao K, An R, Xiang Q, et al. Acid-sensing ion channels regulate nucleus pulposus cell inflammation and pyroptosis via the NLRP3 inflammasome in intervertebral disc degeneration. Cell Prolif. 2021;54(1):e12941. doi:10.1111/cpr.12941

81. Culemann S, Grüneboom A, Nicolás-Ávila JÁ, et al. Locally renewing resident synovial macrophages provide a protective barrier for the joint Nature. 2019;572(7771):670-675.

82. Qian J, Fu P, Li S, et al. miR-107 affects cartilage matrix degradation in the pathogenesis of knee osteoarthritis by regulating caspase-1 J Orthop Surg Res. 2021;16(1):40. doi:10.1186/s13018-020-02121-7

83. Zhang L, Qiu J, Shi J, et al. MicroRNA-140-5p represses chondrocyte pyroptosis and relieves cartilage injury in osteoarthritis by inhibiting cathepsin B/Nod-like receptor protein 3. Bioengineered. 2021;12(2):9949-9964.

84. $\mathrm{Xu} \mathrm{H}, \mathrm{Xu} \mathrm{B}$, Yokota S-I. BMSC-derived exosomes ameliorate osteoarthritis by inhibiting pyroptosis of cartilage via delivering miR-326 targeting HDAC3 and STAT1//NF-kappaB p65 to chondrocytes. Mediators Inflamm. 2021;2021:9972805. doi:10.1155/2021/9972805

85. Castelblanco M, Lugrin J, Ehirchiou D, et al. Hydrogen sulfide inhibits NLRP3 inflammasome activation and reduces cytokine production both in vitro and in a mouse model of inflammation. J Biol Chem. 2018;293(7):2546-2557. doi:10.1074/jbc.M117.806869 
86. Wei Z, Zhan X, Ding K, et al. Dihydrotanshinone I specifically inhibits NLRP3 inflammasome activation and protects against septic shock in vivo. Front Pharmacol. 2021;12:750815.

87. Sarbadhikary P, George BP, Abrahamse H. Inhibitory role of berberine, an isoquinoline alkaloid, on NLRP3 inflammasome activation for the treatment of inflammatory diseases. Molecules. 2021;26(20):6238. doi:10.3390/molecules26206238

88. Liu Q, Wu Z, Hu D, et al. Low dose of indomethacin and Hedgehog signaling inhibitor administration synergistically attenuates cartilage damage in osteoarthritis by controlling chondrocytes pyroptosis. Gene. 2019;712:143959. doi:10.1016/j.gene.2019.143959

89. Zu Y, Mu Y, Li Q, et al. Icariin alleviates osteoarthritis by inhibiting NLRP3-mediated pyroptosis. J Orthop Surg Res. 2019;14(1):307. doi:10.1186/s13018-019-1307-6

90. Hu J, Zhou J, Wu J, et al. Loganin ameliorates cartilage degeneration and osteoarthritis development in an osteoarthritis mouse model through inhibition of NF-kappaB activity and pyroptosis in chondrocytes. $J$ Ethnopharmacol. 2020;247:112261. doi:10.1016/j.jep.2019.112261

91. Kumagai K, Toyoda F, Staunton CA, et al. Activation of a chondrocyte volume-sensitive Cl(-) conductance prior to macroscopic cartilage lesion formation in the rabbit knee anterior cruciate ligament transection osteoarthritis model. Osteoarthr Cartil. 2016;24(10):1786-1794. doi:10.1016/ j.joca.2016.05.019

92. Zhou C, Tang X, Xu J, et al. Opening of the CLC-3 chloride channel induced by dihydroartemisinin contributed to early apoptotic events in human poorly differentiated nasopharyngeal carcinoma cells. J Cell Biochem. 2018;119(11):9560-9572. doi:10.1002/jcb.27274

93. Huang L, Li Y-J, Li -P-P, et al. Aggravated intestinal apoptosis by ClC-3 deletion is lethal to mice endotoxemia. Cell Biol Int. 2018;42 (10): 1445-1453. doi:10.1002/cbin.11025

94. Fan F, Liu T, Wang X, et al. ClC-3 expression and its association with hyperglycemia induced HT22 hippocampal neuronal cell apoptosis. J Diabetes Res. 2016;2016:2984380. doi:10.1155/2016/2984380

95. Bi M, Hong S, Ma LJ, et al. Chloride channel protein 2 prevents glutamate-induced apoptosis in retinal ganglion cells. Iran J Basic Med Sci. 2016;19(7):705-711

96. Deng Z, Li W, Alahdal M, et al. Overexpression of ClC-3 chloride channel in chondrosarcoma: an in vivo immunohistochemical study with tissue microarray. Med Sci Monit. 2019;25:5044-5053. doi:10.12659/MSM.917382

97. Driban JB, Hootman JM, Sitler MR, et al. Is participation in certain sports associated with knee osteoarthritis? a systematic review. $J$ Athl Train. 2017;52(6):497-506. doi:10.4085/1062-6050-50.2.08

98. Zeighami A, Dumas R, Aissaoui R. Knee loading in OA subjects is correlated to flexion and adduction moments and to contact point locations. Sci Rep. 2021;11(1):8594. doi:10.1038/s41598-021-87978-2

99. Miller RH, Krupenevich RL. Medial knee cartilage is unlikely to withstand a lifetime of running without positive adaptation: a theoretical biomechanical model of failure phenomena. PeerJ. 2020;8:e9676. doi:10.7717/peerj.9676

100. Bennell KL, Bowles K-A, Wang Y, et al. Higher dynamic medial knee load predicts greater cartilage loss over 12 months in medial knee osteoarthritis. Ann Rheum Dis. 2011;70(10):1770-1774. doi:10.1136/ard.2010.147082

101. Knowles N, Whittier DE, Besler BA, et al. Proximal tibia bone stiffness and strength in HR-pQCT- and QCT-based finite element models. Ann Biomed Eng. 2021;49(9):2389-2398. doi:10.1007/s10439-021-02789-w

102. Wang H, Wang R, Wang Z, et al. ClC-3 chloride channel functions as a mechanically sensitive channel in osteoblasts. Biochem Cell Biol. 2015;93(6):558-565. doi:10.1139/bcb-2015-0018

103. Harasztosi C, Gummer AW, Acsády L. The chloride-channel blocker 9-anthracenecarboxylic acid reduces the nonlinear capacitance of prestin-associated charge movement. Eur J Neurosci. 2016;43(8):1062-1074. doi:10.1111/ejn.13209

104. Li Z, Huang Z, Zhang H, et al. Moderate-intensity exercise alleviates pyroptosis by promoting autophagy in osteoarthritis via the P2X7/AMPK/ mTOR axis. Cell Death Discov. 2021;7(1):346. doi:10.1038/s41420-021-00746-z

Journal of Inflammation Research

\section{Publish your work in this journal}

The Journal of Inflammation Research is an international, peer-reviewed open-access journal that welcomes laboratory and clinical findings on the molecular basis, cell biology and pharmacology of inflammation including original research, reviews, symposium reports, hypothesis formation and commentaries on: acute/chronic inflammation; mediators of inflammation; cellular processes; molecular mechanisms; pharmacology and novel anti-inflammatory drugs; clinical conditions involving inflammation. The manuscript management system is completely online and includes a very quick and fair peer-review system. Visit http://www.dovepress.com/testimonials.php to read real quotes from published authors.

Submit your manuscript here: https://www.dovepress.com/journal-of-inflammation-research-journal 